\title{
Packing of spanning mixed hyperarborescences with flexible roots via matroid intersection
}

\author{
Florian Hörsch \\ Univ. Grenoble Alpes \\ INP, GSCOP \\ Grenoble, France \\ florian.hoersch@grenoble-inp.fr
}

\author{
Zoltán Szigeti \\ Univ. Grenoble Alpes \\ INP, GSCOP \\ Grenoble, France \\ zoltan.szigeti@grenoble-inp.fr
}

Submitted: Dec 21, 2020; Accepted: Jun 8, 2021; Published: Jul 30, 2021

C) The authors. Released under the CC BY-ND license (International 4.0).

\begin{abstract}
Given a mixed hypergraph $\mathcal{F}=(V, \mathcal{A} \cup \mathcal{E})$, a non-negative integer $k$ and functions $f, g: V \rightarrow \mathbb{Z}_{\geqslant 0}$, a packing of $k$ spanning mixed hyperarborescences of $\mathcal{F}$ is called $(k, f, g)$-flexible if every $v \in V$ is the root of at least $f(v)$ and at most $g(v)$ of the mixed hyperarborescences. We give a characterization of the mixed hypergraphs admitting such packings. This generalizes results of Frank and, more recently, Gao and Yang. Our approach is based on matroid intersection, generalizing a construction of Edmonds. We also obtain an algorithm for finding a minimum weight solution to the problem mentioned above.
\end{abstract}

Mathematics Subject Classifications: 05C40, 05C70, 05C20, 05C65

\section{Introduction}

The purpose of this article is to generalize a recent result of Gao and Yang [12] on packing spanning mixed arborescences with flexible roots from mixed graphs to mixed hypergraphs using matroid intersection. Our approach also yields an algorithm for the weighted version of this problem.

In order to understand the introduction, the reader may find all the necessary definitions in Section 2.

The most basic setting when dealing with packings of spanning arborescences is the following one: Given a directed graph $D=(V, A)$ and a multiset $R$ of vertices in $V$, we want to find a packing $\left\{B_{r}: r \in R\right\}$ of spanning $r$-arborescences of $D$. The following result was proven by Edmonds in 1973 in [3] and is fundamental to the theory of arborescence 
packings. It gives a complete characterization for the existence of packings of spanning arborescences with fixed roots in the basic setting.

Theorem 1 . Let $D=(V, A)$ be a digraph and $R$ a multiset of $V$. There exists a packing $\left\{B_{r}: r \in R\right\}$ of spanning $r$-arborescences in $D$ if and only if $d_{A}^{-}(X) \geqslant|R-X|$ for all $\varnothing \neq$ $X \subseteq V$.

Another celebrated achievement of Edmonds is a connection between the theory of packings of spanning arborescences in digraphs and matroid theory. For that, he considers two matroids on the arc set $A$ of $D$. The first matroid $\mathrm{M}_{1}$ is the $k$-sum of the graphic matroid of the underlying graph of $D$ where $k=|R|$. In other words, an arc set is independent in $\mathrm{M}_{1}$ if and only if the corresponding edge set can be partitioned into $k$ forests. The second matroid $\mathrm{M}_{2}$ is the direct sum of the uniform matroids of rank $k-|R \cap v|$ on the set $\delta_{A}^{-}(v)$ of arcs entering $v$ for all $v \in V$. We refer to these matroids as the $k$-graphic matroid and the $R$-partition matroid of $D$, respectively. Edmonds proved in [4] that the arc sets of the packings of spanning arborescences of $D$ with respect to the root set $R$ are exactly the common independent sets of $\mathrm{M}_{1}$ and $\mathrm{M}_{2}$ of size $k(|V|-1)$. The following observation which can simply be obtained from Theorem 1 is crucial for this modeling. It can be found as Theorem 13.3.20 in [11]. It can also be found as Lemma 5.4.6 in [8], where a direct proof is provided.

Theorem 2. Let $D=(V, A)$ be a digraph and $R$ a multiset of $V$ of size $k$. Some $A^{\prime} \subseteq A$ is the arc set of a packing $\left\{B_{r}: r \in R\right\}$ of spanning $r$-arborescences in $D$ if and only if the underlying edge set of $A^{\prime}$ is the edge set of a packing of $k$ spanning trees of the underlying graph of $D$ and $d_{A^{\prime}}^{-}(v)=k-|R \cap v|$ for all $v \in V$.

A first way of generalizing the results obtained in the basic setting is the consideration of dypergraphs instead of digraphs. The following generalization of Theorem 1 was proved in a stronger form by Frank, Király and Király in [9].

Theorem 3. Let $\mathcal{D}=(V, \mathcal{A})$ be a dypergraph and $R$ a multiset in $V$. There exists a packing $\left\{\mathcal{B}_{r}: r \in R\right\}$ of spanning $r$-hyperarborescences in $\mathcal{D}$ if and only if $d_{\mathcal{A}}^{-}(X) \geqslant|R-X|$ for all $\varnothing \neq X \subseteq V$.

We can conclude the following statement from Theorem 3 using the same technique that was used in [11] to conclude Theorem 2 from Theorem 1. We therefore omit the proof.

Theorem 4 . Let $\mathcal{D}=(V, \mathcal{A})$ be a dypergraph, $k \in \mathbb{Z}_{\geqslant 0}$ and $R$ a multiset in $V$ of size $k$. Some $\mathcal{A}^{\prime} \subseteq \mathcal{A}$ is the dyperedge set of a packing $\left\{\mathcal{B}_{r}: r \in R\right\}$ of spanning $r$-hyperarborescences in $\mathcal{D}$ if and only if the underlying hyperedge set of $\mathcal{A}^{\prime}$ is the hyperedge set of a packing of $k$ spanning hypertrees of the underlying hypergraph of $\mathcal{D}$ and $d_{\mathcal{A}^{\prime}}^{-}(v)=k-|R \cap v|$ for all $v \in V$.

The following algorithmic result has been proven by Fortier et al. in [6].

Theorem 5. Let $\mathcal{D}=(V, \mathcal{A})$ be a dypergraph and $R$ a multiset in $V$ such that $\mathcal{A}$ is the dyperedge set of a packing $\left\{\mathcal{B}_{r}: r \in R\right\}$ of spanning $r$-hyperarborescences in $\mathcal{D}$. Then such a packing can be found in polynomial time. 
The above idea of approaching the problem of packing spanning arborescences via matroid intersection is useful for two reasons. On the one hand, one can apply Edmonds' matroid intersection theorem [2] to get the characterization for the existence of the packing. On the other hand, one can apply Edmonds' weighted matroid intersection algorithm [5] to find a packing of minimum total weight. These results of Edmonds are presented in the following theorem.

Theorem 6. Let $\mathrm{M}_{1}=\left(S, r_{1}\right)$ and $\mathrm{M}_{2}=\left(S, r_{2}\right)$ be two matroids on a common ground set $S$ with polynomial independence oracles for $\mathrm{M}_{1}$ and $\mathrm{M}_{2}$ being available, $\mu \in \mathbb{Z}_{\geqslant 0}$ and $w: S \rightarrow \mathbb{R}$ a weight function.

(a) [2] A common independent set of size $\mu$ of $\mathrm{M}_{1}$ and $\mathrm{M}_{2}$ exists if and only if $r_{1}(Z)+$ $r_{2}(S-Z) \geqslant \mu$ for all $Z \subseteq S$.

(b) [5] We can decide if a common independent set of size $\mu$ of $M_{1}$ and $M_{2}$ exists in polynomial time. Further, if this is the case, then one of minimum weight with respect to $w$ can be computed in polynomial time.

Giving a characterization in terms of matroid intersection for packings of spanning arborescences in a more general setting is the main purpose of this article.

Firstly, the generalization concerns mixed hypergraphs instead of digraphs. Secondly, the generalization relaxes the condition of the roots of the arborescences being fixed. We are given a mixed hypergraph $\mathcal{F}=(\boldsymbol{V}, \mathcal{A} \cup \mathcal{E})$, a non-negative integer $\boldsymbol{k}$ and functions $\boldsymbol{f}, \boldsymbol{g}: V \rightarrow \mathbb{Z}_{\geqslant 0}$, and we want to find a packing of $k$ spanning mixed $r_{i}$-hyperarborescences $\left\{\mathcal{B}_{i}: i \in\{1, \ldots, k\}\right\}$ in $\mathcal{F}$ such that every vertex $v \in V$ is the root of at least $f(v)$ and at most $g(v)$ of the $k$ mixed hyperarborescences. We call such a packing $(k, f, g)$-flexible. The digraphic case of this problem has been successfully treated by Frank in [7]. He proved the following theorem characterizing the digraphs admitting a $(k, f, g)$-flexible packing of spanning arborescences for given $k, f, g$.

Theorem 7 . Let $D=(V, A)$ be a digraph, $k \in \mathbb{Z}_{\geqslant 0}$ and $f, g: V \rightarrow \mathbb{Z}_{\geqslant 0}$ functions. There exists a $(k, f, g)$-flexible packing of spanning arborescences in $D$ if and only if we have

$$
\begin{aligned}
f(v) & \leqslant g(v) & & \text { for every } v \in V, \\
e_{A}(\mathcal{P}) & \geqslant k(|\mathcal{P}|-1)+f(V-\cup \mathcal{P}) & & \text { for every subpartition } \mathcal{P} \text { of } V, \\
d_{A}^{-}(X) & \geqslant k-g(X) & & \text { for every nonempty } X \subseteq V .
\end{aligned}
$$

Further, Frank in [7] gave an algorithm to find such a packing if it exists. Recently, this has been generalized to the case of mixed graphs by Gao and Yang in [12]. Our aim is to extend this latter result to mixed hypergraphs.

The basic insight of our approach is that, given a packing of $k$ spanning hyperarborescences in a dypergraph $\mathcal{D}=(V, \mathcal{A})$, for every vertex $v \in V$, the number of $v$ hyperarborescences in the packing plus the in-degree of $v$ in the packing is equal to $k$. We use this fact to show that $(k, f, g)$-flexible packings of spanning mixed hyperarborescences 
in mixed hypergraphs can be modeled as the intersection of two matroids. While a hypergraphic analogue of the $k$-graphic matroid is maintained as one of the two matroids, the $R$-partition matroid is replaced by a more general object, a so-called generalized partition matroid in which also $f$ and $g$ are encoded. Using Theorem 6(a), this allows to obtain the following characterization for $(k, f, g)$-flexible packings of spanning mixed hyperarborescences in mixed hypergraphs which is the main contribution of this article. It is a literal generalization of the theorem of Gao and Yang to mixed hypergraphs. Our proof is completely different from the one in [12] and works for mixed hypergraphs.

Theorem 8. Let $\mathcal{F}=(V, \mathcal{A} \cup \mathcal{E})$ be a mixed hypergraph, $k \in \mathbb{Z}_{\geqslant 0}$ and $f, g: V \rightarrow \mathbb{Z}_{\geqslant 0}$ functions. There exists a $(k, f, g)$-flexible packing of spanning mixed hyperarborescences in $\mathcal{F}$ if and only if we have

$$
\begin{array}{rlrl}
f(v) & \leqslant g(v) & \text { for every } v \in V, \\
e_{\mathcal{E} \cup \mathcal{A}}(\mathcal{P}) & \geqslant k(|\mathcal{P}|-1)+f(V-\cup \mathcal{P}) & \text { for every subpartition } \mathcal{P} \text { of } V, \\
e_{\mathcal{E} \cup \mathcal{A}}(\mathcal{P}) & \geqslant k|\mathcal{P}|-g(\cup \mathcal{P}) & & \text { for every subpartition } \mathcal{P} \text { of } V .
\end{array}
$$

While the technique of matroid intersection for arborescence packings is routinely used as a tool to obtain algorithms for the weighted cases, we also obtain a new structural result via matroid intersection. By Theorem 6(b), the previous observation also yields an algorithm to compute a $(k, f, g)$-flexible packing of spanning mixed hyperarborescences of minimum total weight in polynomial time.

Theorem 9. Let $\mathcal{F}=(V, \mathcal{A} \cup \mathcal{E})$ be a mixed hypergraph, $k \in \mathbb{Z}_{\geqslant 0}, f, g: V \rightarrow \mathbb{Z}_{\geqslant 0}$ functions and $w: \mathcal{A} \cup \mathcal{E} \rightarrow \mathbb{R}$ a weight function. Then a $(k, f, g)$-flexible packing of spanning mixed hyperarborescences in $\mathcal{F}$ of minimum weight with respect to $w$ can be computed in polynomial time, if there exists one.

\section{Definitions}

A multiset $R$ is a collection of elements in which an element may appear several times. For some $x$, we denote by $|\boldsymbol{R} \cap \boldsymbol{x}|$ the number of times $x$ is contained in $R$. Often, for a single element set $\{s\}$, we use $s$.

For some directed graph (in short, digraph) $D=(V, A)$ and $r \in V$, an $r$-arborescence in $D$ is a subgraph of $D$ whose underlying graph is a tree and in which all the vertices except $r$ have exactly 1 arc entering. An $r$-arborescence of $D$ is called spanning if it contains all the vertices of $D$. By a packing of arborescences we mean a set of arc-disjoint arborescences.

A mixed hypergraph is a tuple $\mathcal{F}=(V, \mathcal{A} \cup \mathcal{E})$ where $\boldsymbol{V}$ is a set of vertices, $\mathcal{A}$ is a set of directed hyperedges (dyperedges) and $\mathcal{E}$ is a set of hyperedges. A dyperedge $\boldsymbol{a}$ is a tuple $(\operatorname{tail}(a)$, head $(a))$ where head(a) is a single vertex in $V$ and $\operatorname{tail}(\boldsymbol{a})$ is a nonempty subset of $V$-head(a) and a hyperedge is a subset of $V$ of size at least two. A mixed hypergraph without hyperedges is a directed hypergraph (dypergraph) and a mixed hypergraph without dyperedges is a hypergraph. We say that $\mathcal{F}$ is a mixed graph if each 
dyperedge has a tail of size exactly one and each hyperedge contains exactly two vertices. A mixed subhypergraph of $\mathcal{F}$ is called spanning if it contains all the vertices of $\mathcal{F}$. For some $\mathcal{E}^{\prime} \subseteq \mathcal{E}$, we denote by $\boldsymbol{V}\left(\mathcal{E}^{\prime}\right)$ the set of vertices in $V$ which are contained in at least one hyperedge in $\mathcal{E}^{\prime}$.

The underlying hypergraph $\mathcal{H}_{\mathcal{F}}=\left(V, \mathcal{E}_{\mathcal{A}} \cup \mathcal{E}\right)$ of $\mathcal{F}$ is obtained from $\mathcal{F}$ by replacing every dyperedge $a \in \mathcal{A}$ by the hyperedge head(a) $\cup \operatorname{tail}(a)$. For a hyperedge $e \in \mathcal{E}$, its corresponding bundle $\mathcal{A}_{e}$ is the set of all possible orientations of $e$, i.e. $\mathcal{A}_{e}=\{(e-v, v)$ : $v \in e\}$. The directed extension $\mathcal{D}_{\mathcal{F}}=\left(V, \mathcal{A} \cup \mathcal{A}_{\mathcal{E}}\right)$ of $\mathcal{F}$ is obtained from $\mathcal{F}$ by replacing every hyperedge in $\mathcal{E}$ by its corresponding bundle, i.e. $\mathcal{A}_{\mathcal{E}}=\cup_{e \in \mathcal{E}} \mathcal{A}_{e}$.

Trimming a dyperedge $a$ means that $a$ is replaced by an arc $u v$ with $v=\operatorname{head}(a)$ and $u \in \operatorname{tail}(a)$. Trimming a hyperedge $e$ means that $e$ is replaced by an arc $u v$ for some $u \neq v \in e$. The mixed hypergraph $\mathcal{F}$ is called a mixed hyperarborescence if its dyperedges and hyperedges can be trimmed to get an arborescence. By a packing of mixed hyperarborescences we mean a set of mixed hyperarborescences whose dyperedge sets and hyperedge sets are pairwise disjoint. A mixed hyperarborescence without hyperedges is called a hyperarborescence. A mixed $r$-hyperarborescence for some $r \in V$ is a mixed hyperarborescence together with a vertex $r$ whose dyperedges and hyperedges can be trimmed to get an $r$-arborescence. A hypergraph is called a hypertree if it can be trimmed to an arborescence.

Given $k \in \mathbb{Z}_{\geqslant 0}$ and $f, g: V \rightarrow \mathbb{Z}_{\geqslant 0}$, we recall that a packing of $k$ spanning mixed $r_{i^{-}}$ hyperarborescences $\left\{\mathcal{B}_{i}: i \in\{1, \ldots, k\}\right\}$ in $\mathcal{F}$ such that every vertex $v \in V$ is the root of at least $f(v)$ and at most $g(v)$ of the $k$ mixed hyperarborescences is called $(k, f, g)$-flexible. Further, a packing of $k$ spanning hyperarborescences in $\mathcal{D}_{\mathcal{F}}$ is called $(k, f, g)$-feasible if every vertex $v \in V$ is the root of at least $f(v)$ and at most $g(v)$ of the $k$ hyperarborescences and for every $e \in \mathcal{E}$, at most one dyperedge of the bundle $\mathcal{A}_{e}$ is contained in the dyperedge set of the packing.

For some $X \subseteq V$, we denote by $\boldsymbol{\delta}_{\mathcal{E}}(\boldsymbol{X})$ the set of hyperedges $e \in \mathcal{E}$ with $e \cap X, e-X \neq$ $\varnothing$ and by $\boldsymbol{\delta}_{\mathcal{A}}^{-}(\boldsymbol{X})$ the set of dyperedges $a \in \mathcal{A}$ with head $(a) \in X$ and $\operatorname{tail}(a)-X \neq \varnothing$. We use $\boldsymbol{d}_{\mathcal{E}}(\boldsymbol{X})$ for $\left|\delta_{\mathcal{E}}(X)\right|$ and $\boldsymbol{d}_{\mathcal{A}}^{-}(\boldsymbol{X})$ for $\left|\delta_{\mathcal{A}}^{-}(X)\right|$. Given a function $m: V \rightarrow \mathbb{R}$ and $X \subseteq V$, we use the notation $\boldsymbol{m}(\boldsymbol{X})=\sum_{v \in X} m(v)$ and hence we consider $m(\varnothing)=0$.

Given a packing $\mathcal{B}=\left\{\mathcal{B}_{r}: r \in R\right\}$ of mixed hyperarborescences, we use $\mathcal{A}(\mathcal{B})$ for $\cup_{r \in R} \mathcal{A}\left(\mathcal{B}_{r}\right)$ and $\mathcal{E}(\mathcal{B})$ for $\cup_{r \in R} \mathcal{E}\left(\mathcal{B}_{r}\right)$. Further, given a weight function $w: \mathcal{A} \cup \mathcal{E} \rightarrow \mathbb{R}$, we use $\boldsymbol{w}(\mathcal{B})$ for $w(\mathcal{A}(\mathcal{B}))+w(\mathcal{E}(\mathcal{B}))$.

Given a subpartition $\mathcal{P}$ of $V$, we use $\mathcal{E}(\mathcal{P})$ for $\cup_{X \in \mathcal{P}} \delta_{\mathcal{E}}(X)$ and $\mathcal{A}(\mathcal{P})$ for $\cup_{X \in \mathcal{P}} \delta_{\mathcal{A}}^{-}(X)$. We use $\boldsymbol{e}_{\mathcal{E}}(\mathcal{P}), \boldsymbol{e}_{\mathcal{A}}(\mathcal{P}), \boldsymbol{e}_{\mathcal{E} \cup \mathcal{A}}(\mathcal{P})$ for the cardinality of $\mathcal{E}(\mathcal{P}), \mathcal{A}(\mathcal{P})$ and $\mathcal{E}(\mathcal{P}) \cup \mathcal{A}(\mathcal{P})$, respectively. Further, we use $\cup \mathcal{P}$ for the union of the classes of $\mathcal{P}$. A hypergraph $\mathcal{H}=$ $(V, \mathcal{E})$ is called partition-connected if $e_{\mathcal{E}}(\mathcal{P}) \geqslant|\mathcal{P}|-1$ for every partition $\mathcal{P}$ of $V$.

Basic notions of matroids which are used in this article can be found in Chapter 5 of [11]. Given a matroid $\mathbf{M}$, we use $\boldsymbol{r}_{\mathbf{M}}$ for the rank function of $\mathbf{M}$.

\section{Relevant matroids}

We now give an overview of the matroids we need for our characterization. 


\subsection{Hypergraphic matroids}

While graphic matroids are well-studied, their generalization to hypergraphs has received significantly less attention. The following matroid construction was first observed by Lorea [13]. Given a hypergraph $\mathcal{H}=(V, \mathcal{E})$, let $\mathcal{I}_{\mathcal{H}}=\left\{\mathcal{Z} \subseteq \mathcal{E}:\left|V\left(\mathcal{Z}^{\prime}\right)\right|>\left|\mathcal{Z}^{\prime}\right|\right.$ for all $\varnothing \neq$ $\left.\mathcal{Z}^{\prime} \subseteq \mathcal{Z}\right\}$

Theorem 10. The set $\mathcal{I}_{\mathcal{H}}$ is the set of independent sets of a matroid $\mathbf{M}_{\mathcal{H}}$ on $\mathcal{E}$.

The matroid $\mathrm{M}_{\mathcal{H}}$ is called the hypergraphic matroid of the hypergraph $\mathcal{H}$.

For our algorithmic result, we need to show that an independence oracle for $\mathrm{M}_{\mathcal{H}}$ exists. In order to do so, we require the following two preliminaries. The first result can be found as Corollary 2.6 in [10].

Proposition 1 . Let $\mathcal{H}=(V, \mathcal{E})$ be a hypergraph. Then $r_{\mathrm{M}_{\mathcal{H}}}(\mathcal{E})=|V|-1$ if and only if $\mathcal{H}$ is partition-connected.

This result is useful due to the next one which can be found in [11] as a comment after Theorem 9.1.22 stating that the proof of Theorem 9.1.15 is algorithmic.

Proposition 2. There is a polynomial time algorithm that decides whether a given hypergraph $\mathcal{H}$ is partition-connected.

We are now ready to conclude that a polynomial time independence oracle for $M_{\mathcal{H}}$ exists. We remark that this is a special case of Problem 13.5.5 in [11].

Lemma 1 . Given a hypergraph $\mathcal{H}=(V, \mathcal{E})$ and $\mathcal{Z} \subseteq \mathcal{E}$, we can decide in polynomial time whether $\mathcal{Z}$ is independent in $\mathrm{M}_{\mathcal{H}}$.

Proof. If $|\mathcal{Z}| \geqslant|V|$, it follows immediately from the definition of $\mathbf{M}_{\mathcal{H}}$ that $\mathcal{Z}$ is dependent in $\mathrm{M}_{\mathcal{H}}$. We may hence suppose that $|\mathcal{Z}| \leqslant|V|-1$. Let a hypergraph $\mathcal{H}^{\prime}$ be obtained from $(V, \mathcal{Z})$ by adding a set $\mathcal{S}$ of $|V|-1-|\mathcal{Z}|$ hyperedges each of which equals $V$. Observe that $|\mathcal{Z} \cup \mathcal{S}|=|V|-1$.

Claim 1. $\mathcal{Z}$ is independent in $\mathbf{M}_{\mathcal{H}}$ if and only if $\mathcal{H}^{\prime}$ is partition-connected.

Proof. First suppose that $\mathcal{Z}$ is independent in $\mathrm{M}_{\mathcal{H}}$. By definition of $\mathrm{M}_{\mathcal{H}}$, for any $\mathcal{Z}^{\prime} \subseteq \mathcal{Z}$, we have $\left|V\left(\mathcal{Z}^{\prime}\right)\right|>\left|\mathcal{Z}^{\prime}\right|$. For any $\mathcal{Z}^{\prime} \subseteq \mathcal{Z} \cup \mathcal{S}$ with $\mathcal{Z}^{\prime} \cap \mathcal{S} \neq \varnothing$, we have $\left|V\left(\mathcal{Z}^{\prime}\right)\right|=|V|>$ $|\mathcal{Z} \cup \mathcal{S}| \geqslant\left|\mathcal{Z}^{\prime}\right|$. This implies that $\left|V\left(\mathcal{Z}^{\prime}\right)\right|>\left|\mathcal{Z}^{\prime}\right|$ for all $\mathcal{Z}^{\prime} \subseteq \mathcal{Z} \cup \mathcal{S}$ and so by definition, $r_{\mathrm{M}_{\mathcal{H}^{\prime}}}(\mathcal{Z} \cup \mathcal{S})=|V|-1$. Now Proposition 1 yields that $\mathcal{H}^{\prime}$ is partition-connected.

Now suppose that $\mathcal{H}^{\prime}$ is partition-connected. It follows from Proposition 1 that $r_{\mathrm{M}_{\mathcal{H}^{\prime}}}(\mathcal{Z} \cup \mathcal{S})=|V|-1=|\mathcal{Z} \cup \mathcal{S}|$. It follows that $\mathrm{M}_{\mathcal{H}^{\prime}}$ is the free matroid, so in particular, $\mathcal{Z}$ is independent in $\mathbf{M}_{\mathcal{H}^{\prime}}$. It follows that $\mathcal{Z}$ is also independent in $\mathbf{M}_{\mathcal{H}}$.

By Claim 1 and as $\mathcal{H}^{\prime}$ can be constructed efficiently, it suffices to check whether $\mathcal{H}^{\prime}$ is partition-connected. By Proposition 2, this can be done in polynomial time.

We also need the $k$-sum matroid of $\mathrm{M}_{\mathcal{H}}$, that is the matroid on ground set $\mathcal{E}$ in which a subset of $\mathcal{E}$ is independent if it can be partitioned into $k$ independent sets of $\mathbf{M}_{\mathcal{H}}$. We call this matroid the $k$-hypergraphic matroid of $\mathcal{H}$ and refer to it as $\mathbf{M}_{\mathcal{H}}^{k}$. The following formula for the rank function of $\mathrm{M}_{\mathcal{H}}^{k}$ was proved by Frank, Király and Kriesell [10]. 
Theorem 11. For all $\mathcal{Z} \subseteq \mathcal{E}$, we have

$$
r_{\mathrm{M}_{\mathcal{H}}^{k}}(\mathcal{Z})=\min \left\{e_{\mathcal{Z}}(\mathcal{P})+k(|V|-|\mathcal{P}|): \mathcal{P} \text { a partition of } V\right\}
$$

We now extend the previous construction to mixed hypergraphs. Let $\mathcal{F}=(V, \mathcal{A} \cup \mathcal{E})$ be a mixed hypergraph, $\mathcal{H}_{\mathcal{F}}=\left(V, \mathcal{E}_{\mathcal{A}} \cup \mathcal{E}\right)$ the underlying hypergraph of $\mathcal{F}$ and $\mathcal{D}_{\mathcal{F}}=$ $\left(V, \mathcal{A} \cup \mathcal{A}_{\mathcal{E}}\right)$ the directed extension of $\mathcal{F}$. We now construct the extended $k$-hypergraphic matroid $\mathbf{M}_{\mathcal{F}}^{k}$ of $\mathcal{F}$ on $\mathcal{A} \cup \mathcal{A}_{\mathcal{E}}$ from $\mathbf{M}_{\mathcal{H}_{\mathcal{F}}}^{k}$ by replacing every $e \in \mathcal{E}$ by $|e|$ parallel copies of itself, associating these elements to the dyperedges in $\mathcal{A}_{e}$ and associating every element of $\mathcal{E}_{\mathcal{A}}$ to the corresponding element in $\mathcal{A}$. We give the following formula for the rank function of $\mathrm{M}_{\mathcal{F}}^{k}$.

Proposition 3. For all $\mathcal{Z} \subseteq \mathcal{A} \cup \mathcal{A}_{\mathcal{E}}$, we have

$r_{\mathrm{M}_{\mathcal{F}}^{k}}(\mathcal{Z})=\min \left\{|\mathcal{Z} \cap \mathcal{A}(\mathcal{P})|+\left|\left\{e \in \mathcal{E}(\mathcal{P}): \mathcal{Z} \cap \mathcal{A}_{e} \neq \varnothing\right\}\right|+k(|V|-|\mathcal{P}|): \mathcal{P}\right.$ a partition of $\left.V\right\}$.

Proof. Let $\mathcal{Z}^{\prime}$ be obtained from $\mathcal{Z}$ by deleting all but one element of $\mathcal{Z} \cap \mathcal{A}_{e}$ for all $e \in \mathcal{E}$ with $\left|\mathcal{Z} \cap \mathcal{A}_{e}\right| \geqslant 2$. As all elements in $\mathcal{A}_{e}$ are parallel in $\mathrm{M}_{\mathcal{F}}^{k}$, we obtain that $r_{\mathrm{M}_{\mathcal{F}}^{k}}(\mathcal{Z})=$ $r_{\mathrm{M}_{\mathcal{F}}^{k}}\left(\mathcal{Z}^{\prime}\right)$. As $\left|\mathcal{Z}^{\prime} \cap \mathcal{A}_{e}\right| \leqslant 1$ for every $e \in \mathcal{E}$, there exists a matroid $\mathrm{M}^{\prime}$ that is isomorphic to $\mathrm{M}_{\mathcal{H}_{\mathcal{F}}}^{k}$, is a restriction of $\mathrm{M}_{\mathcal{F}}^{k}$ and whose ground set contains $\mathcal{Z}^{\prime}$. It follows from Theorem 11 that $r_{\mathrm{M}_{\mathcal{F}}^{k}}(\mathcal{Z})=r_{\mathrm{M}_{\mathcal{F}}^{k}}\left(\mathcal{Z}^{\prime}\right)=r_{\mathrm{M}^{\prime}}\left(\mathcal{Z}^{\prime}\right)=\min \left\{\left|\mathcal{Z}^{\prime} \cap \mathcal{A}(\mathcal{P})\right|+\left|\mathcal{Z}^{\prime} \cap \mathcal{A}_{\mathcal{E}}(\mathcal{P})\right|+k(|V|-|\mathcal{P}|)\right.$ : $\mathcal{P}$ a partition of $V\}=\min \left\{|\mathcal{Z} \cap \mathcal{A}(\mathcal{P})|+\left|\left\{e \in \mathcal{E}(\mathcal{P}): \mathcal{Z} \cap \mathcal{A}_{e} \neq \varnothing\right\}\right|+k(|V|-|\mathcal{P}|)\right.$ : $\mathcal{P}$ a partition of $V\}$.

Again, for the algorithmic part, we need to show that an independence oracle for $\mathrm{M}_{\mathcal{F}}^{k}$ is available. We need the following preliminary result on matroids which was proven by Edmonds [1].

Proposition 4. Let $\mathrm{M}$ be a matroid such that a polynomial time independence oracle for $\mathrm{M}$ is available and $k$ a positive integer. Then a polynomial time independence oracle for the $k$-sum of $\mathrm{M}$ is also available.

Lemma 2. Given a mixed hypergraph $\mathcal{F}=(V, \mathcal{A} \cup \mathcal{E})$ and $\mathcal{Z} \subseteq \mathcal{A} \cup \mathcal{A}_{\mathcal{E}}$, we can decide in polynomial time if $\mathcal{Z}$ is independent in $\mathrm{M}_{\mathcal{F}}^{k}$.

Proof. If $\mathcal{Z}$ contains at least 2 elements of $\mathcal{A}_{e}$ for some $e \in \mathcal{E}$, then $\mathcal{Z}$ is dependent in $\mathrm{M}_{\mathcal{F}}^{k}$ by definition. Otherwise, there is a matroid $\mathrm{M}^{\prime}$ that is isomorphic to $\mathrm{M}_{\mathcal{H}_{\mathcal{F}}}^{k}$, is a restriction of $\mathrm{M}_{\mathcal{F}}^{k}$ and whose ground set contains $\mathcal{Z}$. Further, $\mathrm{M}^{\prime}$ can be found efficiently. It therefore suffices to prove that a polynomial time independence oracle for $\mathrm{M}_{\mathcal{H}_{\mathcal{F}}}^{k}$ is available. This follows immediately from Lemma 1 and Proposition 4.

\subsection{Generalized partition matroids}

The other matroid we consider is called a generalized partition matroid and plays the role of the $R$-partition matroid. It has been considered in a slightly weaker form in [11], see Problem 5.3.4. 
Let $\left\{\boldsymbol{S}_{\mathbf{1}}, \ldots, \boldsymbol{S}_{\boldsymbol{n}}\right\}$ be a partition of a set $\boldsymbol{S}$ and $\boldsymbol{\mu}, \boldsymbol{\alpha}_{\boldsymbol{i}}, \boldsymbol{\beta}_{\boldsymbol{i}} \in \mathbb{Z}$ for all $i \in\{1, \ldots, n\}$. For $Y, Z, \ldots \subseteq S$ and $i \in\{1, \ldots, n\}$, let $\boldsymbol{y}_{\boldsymbol{i}}=\left|Y \cap S_{i}\right|, \boldsymbol{z}_{\boldsymbol{i}}=\left|Z \cap S_{i}\right|$ etc. Let

$$
\begin{aligned}
& \mathcal{I}=\left\{Z \subseteq S: z_{i} \leqslant \beta_{i} \text { for all } i \in\{1, \ldots, n\}, \sum_{i=1}^{n} \max \left\{\alpha_{i}, z_{i}\right\} \leqslant \mu\right\}, \\
& \mathcal{B}=\left\{Z \subseteq S: \alpha_{i} \leqslant z_{i} \leqslant \beta_{i} \text { for all } i \in\{1, \ldots, n\},|Z|=\mu\right\} \text { and } \\
& \boldsymbol{r}(\boldsymbol{Z})=\min \left\{\sum_{i=1}^{n} \min \left\{\beta_{i}, z_{i}\right\}, \mu-\sum_{i=1}^{n} \max \left\{\alpha_{i}-z_{i}, 0\right\}\right\} \text { for all } Z \subseteq S .
\end{aligned}
$$

We acknowledge that there is a different proof of the following result which is shorter but involves some sophisticated theory on generalized polymatroids, see [14].

Theorem 12. There exists a matroid $M$ whose set of independent sets is $\mathcal{I}$, set of bases is $\mathcal{B}$ and rank function is $r$ if and only if

$$
\begin{aligned}
& \max \left\{\alpha_{i}, 0\right\} \leqslant \min \left\{\beta_{i}, s_{i}\right\} \text { for all } i \in\{1, \ldots, n\}, \\
& \sum_{i=1}^{n} \max \left\{\alpha_{i}, 0\right\} \leqslant \mu \leqslant \sum_{i=1}^{n} \min \left\{\beta_{i}, s_{i}\right\} .
\end{aligned}
$$

The matroid $\mathrm{M}$ in Theorem 12 is called generalized partition matroid.

Proof. First suppose that there exists such a matroid $\mathrm{M}$ and let $Z \in \mathcal{B}$. For all $i \in$ $\{1, \ldots, n\}$, this yields $\max \left\{\alpha_{i}, 0\right\} \leqslant z_{i} \leqslant \min \left\{\beta_{i}, s_{i}\right\}$, so (5) holds. Further, we obtain $\sum_{i=1}^{n} \max \left\{\alpha_{i}, 0\right\} \leqslant \sum_{i=1}^{n} z_{i}=|Z|=\mu$ and $\sum_{i=1}^{n} \max \left\{\beta_{i}, s_{i}\right\} \geqslant \sum_{i=1}^{n} z_{i}=|Z|=\mu$, so (6) holds.

We now show sufficiency through three claims.

Claim 2. $\mathcal{I}$ forms the collection of independent sets of a matroid $\mathbf{M}$, i.e. $\mathcal{I}$ satisfies the following 3 independence axioms:

$\left(I_{0}\right) \varnothing \in \mathcal{I}$

$\left(I_{1}\right)$ if $Z \subset Z^{\prime}$ and $Z^{\prime} \in \mathcal{I}$, then $Z \in \mathcal{I}$,

$\left(I_{2}\right)$ if $Z, Z^{\prime} \in \mathcal{I}$ and $|Z|<\left|Z^{\prime}\right|$, then there exists some $x \in Z^{\prime}-Z$ such that $Z \cup\{x\} \in \mathcal{I}$.

Proof. $\left(I_{0}\right)$ : By (5), we have $\beta_{i} \geqslant 0=\left|\varnothing \cap S_{i}\right|$ for $i \in\{1, \ldots, n\}$. Further, (6) yields $\sum_{i=1}^{n} \max \left\{\alpha_{i},\left|\varnothing \cap S_{i}\right|\right\}=\sum_{i=1}^{n} \max \left\{\alpha_{i}, 0\right\} \leqslant \mu$. This yields $\varnothing \in \mathcal{I}$.

$\left(I_{1}\right)$ : Let $\boldsymbol{Z} \subset \boldsymbol{Z}^{\prime} \in \mathcal{I}$. Then $z_{i} \leqslant z_{i}^{\prime} \leqslant \beta_{i}$ for $i=1, \ldots, n$ and $\sum_{i=1}^{n} \max \left\{\alpha_{i}, z_{i}\right\} \leqslant$ $\sum_{i=1}^{n} \max \left\{\alpha_{i}, z_{i}^{\prime}\right\} \leqslant \mu$, so we have $Z \in \mathcal{I}$.

$\left(I_{2}\right)$ : Let $\boldsymbol{Z}, \boldsymbol{Z}^{\prime} \in \mathcal{I}$ and $|Z|<\left|Z^{\prime}\right|$. Let $\boldsymbol{J}=\left\{j \in\{1, \ldots, n\}: z_{j}<z_{j}^{\prime}\right\}$. Observe that $J \neq \varnothing$ as $|Z|<\left|Z^{\prime}\right|$. For all $j \in J$, let $\boldsymbol{x}_{\boldsymbol{j}} \in\left(S_{j} \cap Z^{\prime}\right)-Z$ and $\boldsymbol{Z}^{\boldsymbol{j}}=Z \cup\left\{x_{j}\right\}$. Observe that for all $j \in J$, we have $z_{j}^{j} \leqslant z_{j}^{\prime} \leqslant \beta_{j}$ and $z_{i}^{j}=z_{i} \leqslant \beta_{i}$ for all $i \in\{1, \ldots, n\}-\{j\}$. In order to prove that $Z^{j} \in \mathcal{I}$ for some $j \in J$, it remains to show that there is some $j \in J$ with $\sum_{i=1}^{n} \max \left\{\alpha_{i}, z_{i}^{j}\right\} \leqslant \mu$. If there is some $j \in J$ with $z_{j}<\alpha_{j}$, then $\sum_{i=1}^{n} \max \left\{\alpha_{i}, z_{i}^{j}\right\}=$ $\sum_{i=1}^{n} \max \left\{\alpha_{i}, z_{i}\right\} \leqslant \mu$, so we are done. We may hence suppose that $z_{j} \geqslant \alpha_{j}$ for all $j \in J$. This yields $\max \left\{\alpha_{j}-z_{j}^{\prime}, 0\right\} \geqslant 0=\max \left\{\alpha_{j}-z_{j}, 0\right\}$ for all $j \in J$. For all 
$i \in\{1, \ldots, n\}-J$, we have $z_{i} \geqslant z_{i}^{\prime}$ yielding $\max \left\{\alpha_{i}-z_{i}^{\prime}, 0\right\} \geqslant \max \left\{\alpha_{i}-z_{i}, 0\right\}$. It follows that $\max \left\{\alpha_{i}-z_{i}^{\prime}, 0\right\} \geqslant \max \left\{\alpha_{i}-z_{i}, 0\right\}$ for all $i \in\{1, \ldots, n\}$. Then, as $|Z|<\left|Z^{\prime}\right|$ and $Z^{\prime} \in \mathcal{I}$, for some arbitrary $j \in J$, we obtain

$$
\begin{aligned}
\sum_{i=1}^{n} \max \left\{\alpha_{i}, z_{i}^{j}\right\} & \leqslant \sum_{i=1}^{n} \max \left\{\alpha_{i}, z_{i}\right\}+1 \\
& =\sum_{i=1}^{n} \max \left\{\alpha_{i}-z_{i}, 0\right\}+\sum_{i=1}^{n} z_{i}+1 \\
& \leqslant \sum_{i=1}^{n} \max \left\{\alpha_{i}-z_{i}^{\prime}, 0\right\}+\sum_{i=1}^{n} z_{i}^{\prime} \\
& =\sum_{i=1}^{n} \max \left\{\alpha_{i}, z_{i}^{\prime}\right\} \\
& \leqslant \mu .
\end{aligned}
$$

Claim 3. $Z \in \mathcal{B}$ if and only if $Z$ is a maximal element in $\mathcal{I}$.

Proof. First let $\boldsymbol{Z} \in \mathcal{B}$. We obtain $\mu=|Z|=\sum_{i=1}^{n} z_{i}=\sum_{i=1}^{n} \max \left\{\alpha_{i}, z_{i}\right\}$. As $\beta_{i} \geqslant z_{i}$ for all $i \in\{1, \ldots, n\}$, we have $Z \in \mathcal{I}$. Further, for any proper superset $Z^{\prime}$ of $Z$, we have $\sum_{i=1}^{n} \max \left\{\alpha_{i}, z_{i}^{\prime}\right\} \geqslant\left|Z^{\prime}\right|>|Z|=\mu$, so $Z^{\prime} \notin \mathcal{I}$. It follows that $Z$ is a maximal element in

Now let $\boldsymbol{Z}$ be a maximal element in $\mathcal{I}$. If $z_{j}<\alpha_{j}$ for some $j \in\{1, \ldots, n\}$, let $\boldsymbol{x}$ $\in S_{j}-Z$ and let $Z^{\prime}=Z \cup\{x\}$. As $\alpha_{j} \leqslant \beta_{j}$ and $Z \in \mathcal{I}$, we obtain $z_{j}^{\prime} \leqslant z_{j}+1 \leqslant \alpha_{j} \leqslant \beta_{j}$ and $z_{i}^{\prime}=z_{i} \leqslant \beta_{i}$ for all $i \in\{1, \ldots, n\}-\{j\}$. Moreover, we have $\sum_{i=1}^{n} \max \left\{\alpha_{i}, z_{i}^{\prime}\right\}=$ $\sum_{i=1}^{n} \max \left\{\alpha_{i}, z_{i}\right\} \leqslant \mu$, so $Z^{\prime} \in \mathcal{I}$. This contradicts the maximality of $Z$. We obtain that $z_{j} \geqslant \alpha_{j}$ for all $j \in\{1, \ldots, n\}$.

As $Z \in \mathcal{I}$, we have $|Z| \leqslant \mu$. If $|Z|<\mu$, by $\sum_{i=1}^{n} \min \left\{\beta_{i}, s_{i}\right\} \geqslant \mu$, there exists some $j \in\{1, \ldots, n\}$ such that $z_{j}<\min \left\{\beta_{j}, s_{j}\right\}$. Let $\boldsymbol{x} \in S_{j}-Z$ and let $\boldsymbol{Z}^{\prime}=Z \cup\{x\}$. We have $z_{j}^{\prime} \leqslant z_{j}+1 \leqslant \beta_{j}$ and $z_{i}^{\prime}=z_{i} \leqslant \beta_{i}$ for all $i \in\{1, \ldots, n\}-\{j\}$. Further, we have $\sum_{i=1}^{n} \max \left\{\alpha_{i}, z_{i}^{\prime}\right\}=\sum_{i=1}^{n} \max \left\{\alpha_{i}, z_{i}\right\}+1=|Z|+1 \leqslant \mu$, so $Z^{\prime} \in \mathcal{I}$. This contradicts the maximality of $Z$. It follows that $|Z|=\mu$, so $Z \in \mathcal{B}$.

Claim 4. The rank function of $\mathrm{M}$ is $r$.

Proof. Let $\boldsymbol{Z} \subseteq S$ and $\boldsymbol{Y}$ be a maximal element of $\mathcal{I}$ in $Z$.

As $Y \in \mathcal{I}$ and $Y \subseteq Z$, we obtain $y_{i} \leqslant \min \left\{\beta_{i}, z_{i}\right\}$ for all $i \in\{1, \ldots, n\}$. This yields $\sum_{i=1}^{n} y_{i} \leqslant \sum_{i=1}^{n} \min \left\{\beta_{i}, z_{i}\right\}$. Further, as $y_{i} \leqslant z_{i}$ for all $i \in\{1, \ldots, n\}$ and $Y \in \mathcal{I}$, we obtain $\sum_{i=1}^{n} y_{i}+\sum_{i=1}^{n} \max \left\{\alpha_{i}-z_{i}, 0\right\}=\sum_{i=1}^{n} \max \left\{\alpha_{i}-z_{i}+y_{i}, y_{i}\right\} \leqslant \sum_{i=1}^{n} \max \left\{\alpha_{i}, y_{i}\right\} \leqslant \mu$, so $\sum_{i=1}^{n} y_{i} \leqslant \mu-\sum_{i=1}^{n} \max \left\{\alpha_{i}-z_{i}, 0\right\}$. It follows that $r_{\mathrm{M}}(Z)=|Y|=\sum_{i=1}^{n} y_{i} \leqslant$ $\min \left\{\sum_{i=1}^{n} \min \left\{\beta_{i}, z_{i}\right\}, \mu-\sum_{i=1}^{n} \max \left\{\alpha_{i}-z_{i}, 0\right\}\right\}=r(Z)$.

Let $\boldsymbol{J}=\left\{j \in\{1, \ldots, n\}: y_{j}<\min \left\{\beta_{j}, z_{j}\right\}\right\}$. If $J=\varnothing$, then we obtain $r_{\mathrm{M}}(Z)=|Y|=$ $\sum_{i=1}^{n} y_{i} \geqslant \sum_{i=1}^{n} \min \left\{\beta_{i}, z_{i}\right\} \geqslant \min \left\{\sum_{i=1}^{n} \min \left\{\beta_{i}, z_{i}\right\}, \mu-\sum_{i=1}^{n} \max \left\{\alpha_{i}-z_{i}, 0\right\}\right\}=r(Z)$, so we are done. We may therefore suppose that $J \neq \varnothing$. 
For all $j \in J$, let $\boldsymbol{x}_{j} \in\left(S_{j} \cap Z\right)-Y$ and let $\boldsymbol{Y}^{j}=Y \cup\left\{x_{j}\right\}$. Note that $Y^{j} \subseteq Z$. For all $j \in J$, we have $y_{j}^{j}=y_{j}+1 \leqslant \beta_{j}$ and $y_{i}^{j}=y_{i} \leqslant \beta_{i}$ for all $i \in\{1, \ldots, n\}-\{j\}$. If $\sum_{i=1}^{n} \max \left\{\alpha_{i}, y_{i}\right\}<\mu$, then for some arbitrary $j \in J$, we have $\sum_{i=1}^{n} \max \left\{\alpha_{i}, y_{i}^{j}\right\} \leqslant$ $\sum_{i=1}^{n} \max \left\{\alpha_{i}, y_{i}\right\}+1 \leqslant \mu$, so we get $Y^{j} \in \mathcal{I}$, a contradiction to the maximality of $Y$. By $Y \in \mathcal{I}$, this yields $\sum_{i=1}^{n} \max \left\{\alpha_{i}, y_{i}\right\}=\mu$.

If there is some $j \in J$ such that $y_{j}<\alpha_{j}$, then $\sum_{i=1}^{n} \max \left\{\alpha_{i}, y_{i}^{j}\right\}=\sum_{i=1}^{n} \max \left\{\alpha_{i}, y_{i}\right\}=$ $\mu$, so we get $Y^{j} \in \mathcal{I}$, a contradiction to the maximality of $Y$. This yields $y_{j} \geqslant \alpha_{j}$ for all $j \in J$, so, as $y_{j} \leqslant z_{j}$, we obtain $\max \left\{\alpha_{j}-y_{j}, 0\right\}=0=\max \left\{\alpha_{j}-z_{j}, 0\right\}$. For all $i \in\{1, \ldots, n\}-J$, we have either $y_{i}=\beta_{i}$ or $y_{i}=z_{i}$. If $y_{i}=\beta_{i}$, by $y_{i} \leqslant z_{i}$ and $\alpha_{i} \leqslant \beta_{i}$, we obtain $\max \left\{\alpha_{i}-y_{i}, 0\right\}=0=\max \left\{\alpha_{i}-z_{i}, 0\right\}$. If $y_{i}=z_{i}$, we clearly obtain $\max \left\{\alpha_{i}-y_{i}, 0\right\}=\max \left\{\alpha_{i}-z_{i}, 0\right\}$. It follows that $\max \left\{\alpha_{i}-y_{i}, 0\right\}=\max \left\{\alpha_{i}-z_{i}, 0\right\}$ holds for all $i \in\{1, \ldots, n\}$.

This yields $r_{\mathrm{M}}(Z)=|Y|=\sum_{i=1}^{n} y_{i}=\sum_{i=1}^{n} \max \left\{\alpha_{i}, y_{i}\right\}-\sum_{i=1}^{n} \max \left\{\alpha_{i}-y_{i}, 0\right\}=$ $\mu-\sum_{i=1}^{n} \max \left\{\alpha_{i}-z_{i}, 0\right\} \geqslant \min \left\{\sum_{i=1}^{n} \min \left\{\beta_{i}, z_{i}\right\}, \mu-\sum_{i=1}^{n} \max \left\{\alpha_{i}-z_{i}, 0\right\}\right\}=r(Z)$.

The three previous claims yield the theorem.

Corollary 1 . Let $\mathcal{D}=\left(V, \mathcal{A}^{\prime}\right)$ be a dypergraph, $k \in \mathbb{Z}_{\geqslant 0}$ and $f, g: V \rightarrow \mathbb{Z}_{\geqslant 0}$ functions such that the following two conditions are satisfied:

$$
\begin{aligned}
\max \{k-g(v), 0\} & \leqslant \min \left\{k-f(v), d_{\mathcal{A}^{\prime}}^{-}(v)\right\} \quad \text { for all } v \in V, \\
\sum_{v \in V} \max \{k-g(v), 0\} & \leqslant k(|V|-1) \leqslant \sum_{v \in V} \min \left\{k-f(v), d_{\mathcal{A}^{\prime}}^{-}(v)\right\} .
\end{aligned}
$$

Then $\left\{\mathcal{Z} \subseteq \mathcal{A}^{\prime}:|\mathcal{Z}|=k(|V|-1), f(v) \leqslant k-d_{\mathcal{Z}}^{-}(v) \leqslant g(v)\right.$ for all $\left.v \in V\right\}$ is the set of bases of a matroid $\mathbf{M}_{\mathcal{D}}^{(\boldsymbol{k}, \boldsymbol{f}, \boldsymbol{g})}$ on $\mathcal{A}^{\prime}$ with rank function

$r_{\mathrm{M}_{\mathcal{D}}^{(k, f, g)}}(\mathcal{Z})=\min \left\{\sum_{v \in V} \min \left\{k-f(v), d_{\mathcal{Z}}^{-}(v)\right\}, k(|V|-1)-\sum_{v \in V} \max \left\{k-g(v)-d_{\mathcal{Z}}^{-}(v), 0\right\}\right\}$.

Proof. Let $\boldsymbol{S}=\mathcal{A}^{\prime}, \boldsymbol{\mu}=k(|V|-1)$ and $\boldsymbol{S}_{\boldsymbol{v}}=\delta_{\mathcal{A}^{\prime}}^{-}(v), \boldsymbol{\alpha}_{\boldsymbol{v}}=k-g(v)$ and $\boldsymbol{\beta}_{\boldsymbol{v}}=k-f(v)$ for all $v \in V$. Then (7) and (8) coincide with (5) and (6), respectively. We can therefore apply Theorem 12 from which the statement follows immediately.

The following is an immediate corollary of the definition of $\mathrm{M}_{\mathcal{D}}^{(k, f, g)}$.

Lemma 3. Given a dypergraph $\mathcal{D}=\left(V, \mathcal{A}^{\prime}\right), k \in \mathbb{Z}_{\geqslant 0}$, functions $f, g: V \rightarrow \mathbb{Z}_{\geqslant 0}$ satisfying (7) and (8) and $\mathcal{Z} \subseteq \mathcal{A}^{\prime}$, we can decide in polynomial time if $\mathcal{Z}$ is independent in $\mathrm{M}_{\mathcal{D}}^{(k, f, g)}$.

\section{Flexible packings by matroid intersection}

We are now ready to show how to model packings of spanning mixed hyperarborescences with flexible roots via matroid intersection.

The following observation allows us to reduce the problem of finding flexible packings of spanning mixed hyperarborescences in a mixed hypergraph to finding feasible packings of spanning hyperarborescences in its directed extension. 
Lemma 4. Let $\mathcal{F}=(V, \mathcal{A} \cup \mathcal{E})$ be a mixed hypergraph, $\mathcal{D}_{\mathcal{F}}=\left(V, \mathcal{A} \cup \mathcal{A}_{\mathcal{E}}\right)$ its directed extension, $k \in \mathbb{Z}_{\geqslant 0}$ and $f, g: V \rightarrow \mathbb{Z}_{\geqslant 0}$ functions. Then there exists a $(k, f, g)$-flexible packing of spanning mixed hyperarborescences of $\mathcal{F}$ if and only if there exists a $(k, f, g)$ feasible packing of spanning hyperarborescences of $\mathcal{D}_{\mathcal{F}}$.

Proof. First suppose that there exists a $(k, f, g)$-flexible packing $\mathcal{B}=\left\{\boldsymbol{B}_{\boldsymbol{i}}: i \in\{1, \ldots, k\}\right\}$ of spanning mixed hyperarborescences of $\mathcal{F}$. Then there is a $(k, f, g)$-flexible packing $\boldsymbol{B}$ $=\left\{\boldsymbol{B}_{\boldsymbol{i}}: i \in\{1, \ldots, k\}\right\}$ of spanning arborescences on $V$ such that $B_{i}$ is a trimming of $\mathcal{B}_{i}$ for all $i \in\{1, \ldots, k\}$. For every $e \in \mathcal{E}(\mathcal{B})$, let $v_{e}$ be the head of the arc to which $e$ is trimmed in $B$ and let $\vec{e} \in \mathcal{A}_{e}$ be the orientation of $e$ where $v_{e}$ is chosen to be its head. Then the set of dyperedges $\mathcal{A}(\mathcal{B}) \cup\{\vec{e}: e \in \mathcal{E}(\mathcal{B})\}$ can be trimmed to $B$ and it contains at most one dyperedge of $\mathcal{A}_{e}$ for all $e \in \mathcal{E}$. It follows that there exists a $(k, f, g)$-feasible packing of spanning hyperarborescences of $\mathcal{D}_{\mathcal{F}}$.

Now suppose that there exists a $(k, f, g)$-feasible packing $\mathcal{B}$ of spanning hyperarborescences of $\mathcal{D}_{\mathcal{F}}$. By definition, $\mathcal{A}_{\mathcal{E}}(\mathcal{B})$ contains at most one dyperedge in $\mathcal{A}_{e}$ for all $e \in \mathcal{E}$. Replacing a dyperedge in $\mathcal{A}_{e}(\mathcal{B})$ by $e$ for all $e \in \mathcal{E}$, we obtain the dyper- and hyperedge set of a $(k, f, g)$-flexible packing of spanning mixed hyperarborescences of $\mathcal{F}$.

We now give the characterization via matroid intersection which is the core of this article.

Theorem 13. Let $\mathcal{F}=(V, \mathcal{A} \cup \mathcal{E})$ be a mixed hypergraph, $\mathcal{D}_{\mathcal{F}}=\left(V, \mathcal{A}^{\prime}=\mathcal{A} \cup \mathcal{A}_{\mathcal{E}}\right)$ its directed extension, $k \in \mathbb{Z}_{\geqslant 0}$ and $f, g: V \rightarrow \mathbb{Z}_{\geqslant 0}$ functions. Suppose that (7) and (8) are satisfied. Then the dyperedge sets of the $(k, f, g)$-feasible packings of spanning hyperarborescences in $\mathcal{D}_{\mathcal{F}}$ are exactly the common independent sets of size $k(|V|-1)$ of $\mathrm{M}_{\mathcal{F}}^{k}$ and $\mathrm{M}_{\mathcal{D}_{\mathcal{F}}}^{(k, f, g)}$.

Proof. For the sake of simplicity, we use $\mathbf{M}_{\mathbf{1}}$ and $\mathbf{M}_{\mathbf{2}}$ for $\mathbf{M}_{\mathcal{F}}^{k}$ and $\mathbf{M}_{\mathcal{D}_{\mathcal{F}}}^{(k, f, g)}$, respectively. As (7) and (8) are satisfied, Corollary 1 yields that $M_{2}$ is well-defined. First, let $\mathcal{Z}$ be the dyperedge set of a $(k, f, g)$-feasible packing of spanning hyperarborescences in $\mathcal{D}_{\mathcal{F}}$. Then the underlying hypergraph of $(V, \mathcal{Z})$ is the union of $k$ hyperedge-disjoint spanning hypertrees of $\mathcal{H}_{\mathcal{F}}$ and $\mathcal{Z}$ contains at most one dyperedge of the bundle $A_{e}$ for all $e \in \mathcal{E}$. It follows that $\mathcal{Z}$ is an independent set of $\mathrm{M}_{1}$. As $\mathcal{Z}$ is the dyperedge set of a packing of $k$ spanning hyperarborescences of $\mathcal{D}_{\mathcal{F}}$, we have $|\mathcal{Z}|=k(|V|-1)$. Since the packing is $(k, f, g)$-feasible, every vertex $v$ is the root of at least $f(v)$ and at most $g(v)$ of the $k$ spanning hyperarborescences of the packing. It follows that $k-g(v) \leqslant d_{\mathcal{Z}}^{-}(v) \leqslant k-f(v)$, so $\mathcal{Z}$ is a basis of $M_{2}$ and therefore an independent set of $M_{2}$.

Now let $\mathcal{Z}$ be a common independent set of $\mathrm{M}_{1}$ and $\mathrm{M}_{2}$ of size $k(|V|-1)$. Then the underlying hypergraph of $(V, \mathcal{Z})$ is the union of $k$ hyperedge-disjoint spanning hypertrees of $\mathcal{H}_{\mathcal{F}}$ and, by $f \geqslant 0$, we have $d_{\mathcal{Z}}^{-}(v) \leqslant k-f(v) \leqslant k$ for all $v \in V$. Let $\boldsymbol{R}$ be the multiset in $V$ in which every vertex $v$ in $V$ is contained $k-d_{\mathcal{Z}}^{-}(v)$ times. By the above, this value is nonnegative for all $v \in V$, so $R$ is well-defined. As $|\mathcal{Z}|=k(|V|-1)$, we have $|R|=\sum_{v \in V}\left(k-d_{\mathcal{Z}}^{-}(v)\right)=k|V|-|\mathcal{Z}|=k|V|-k(|V|-1)=k$. Also, by construction $d_{\mathcal{Z}}^{-}(v)=k-|R \cap v|$ for all $v \in V$. Theorem 4 therefore implies that $\mathcal{Z}$ is the set of 
dyperedges of a packing of $k$ spanning hyperarborescences of $\mathcal{D}_{\mathcal{F}}$ with root set $R$, so each $v \in V$ is the root of $k-d_{\mathcal{Z}}^{-}(v)$ of them. As $\mathcal{Z}$ is an independent set of $\mathrm{M}_{2}$, we obtain that $k-g(v) \leqslant d_{\mathcal{Z}}^{-}(v) \leqslant k-f(v)$, so $f(v) \leqslant k-d_{\mathcal{Z}}^{-}(v) \leqslant g(v)$. Finally, as $\mathcal{Z}$ is independent in $\mathrm{M}_{1}, \mathcal{Z}$ contains at most one dyperedge of the bundle $A_{e}$ for all $e \in \mathcal{E}$. It follows that $\mathcal{Z}$ is the dyperedge set of a $(k, f, g)$-feasible packing of spanning hyperarborescences in $\mathcal{D}_{\mathcal{F}}$.

\section{The proofs of Theorems 8 and 9}

This section is dedicated to concluding Theorems 8 and 9 from the matroidal characterization presented in Theorem 13. We first need two slightly technical lemmas. The first one shows the necessity in Theorem 8 and is also used in the proof of Theorem 9 .

Lemma 5. Let $\mathcal{F}=(V, \mathcal{A} \cup \mathcal{E})$ be a mixed hypergraph, $k \in \mathbb{Z}_{\geqslant 0}$ and $f, g: V \rightarrow \mathbb{Z}_{\geqslant 0}$ functions. If there exists a $(k, f, g)$-flexible packing of spanning mixed hyperarborescences in $\mathcal{F}$, then (1), (2) and (3) are satisfied.

Proof. By Lemma 4, there exists a $(k, f, g)$-feasible packing $\mathcal{B}=\left\{\boldsymbol{B}_{\boldsymbol{i}}: i \in\{1, \ldots, k\}\right\}$ of spanning hyperarborescences in $\mathcal{D}_{\mathcal{F}}$. Let $\boldsymbol{s}(\boldsymbol{X})$ denote the number of hyperarborescences in $\mathcal{B}$ whose roots are in the vertex set $X$. Then for every vertex $v \in V, f(v) \leqslant s(v) \leqslant g(v)$ and hence (1) is satisfied. Let now $\mathcal{P}$ be a subpartition of $V$. Let $\mathcal{Z}$ be the dyperedge set of $\mathcal{B}$. By definition, $\mathcal{Z}$ contains at most one dyperedge in $\mathcal{A}_{e}$ for all $e \in \mathcal{E}$. It follows that $e_{\mathcal{E} \cup \mathcal{A}}(\mathcal{P}) \geqslant \sum_{X \in \mathcal{P}} d_{\mathcal{Z}}^{-}(X)$. Since $\mathcal{B}$ is a packing of $k$ spanning hyperarborescences of $\mathcal{D}_{\mathcal{F}}$, we have $d_{\mathcal{Z}}^{-}(X) \geqslant k-s(X)$. Thus $e_{\mathcal{E} \cup \mathcal{A}}(\mathcal{P}) \geqslant k|\mathcal{P}|-s(\cup \mathcal{P})$. Since $s(V)=k$ and $f(v) \leqslant s(v) \leqslant g(v)$ for all $v \in V$, we have $s(\cup \mathcal{P})=s(V)-s(V-\cup \mathcal{P}) \leqslant k-f(V-\cup \mathcal{P})$, yielding (2). Further, we have $s(\cup \mathcal{P}) \leqslant g(\cup \mathcal{P})$ yielding (3).

The second one allows us to use Theorem 13 in the proofs of Theorems 8 and 9 .

Lemma 6 . Let $\mathcal{F}=(V, \mathcal{A} \cup \mathcal{E})$ be a mixed hypergraph, $\mathcal{D}_{\mathcal{F}}=\left(V, \mathcal{A}^{\prime}=\mathcal{A} \cup \mathcal{A}_{\mathcal{E}}\right)$ its directed extension, $k \in \mathbb{Z}_{\geqslant 0}$ and $f, g: V \rightarrow \mathbb{Z}_{\geqslant 0}$ functions. If (1), (2) and (3) are satisfied, then (7) and (8) are satisfied.

Proof. By (2) for $\mathcal{P}=\varnothing$ and $f \geqslant 0$, we obtain $k=-k(|\varnothing|-1) \geqslant f(V-\varnothing)-e_{\mathcal{E} \cup \mathcal{A}}(\varnothing)=$ $f(V)$. By (3) for $\mathcal{P}=\{V\}$, it follows that $k=k|\{V\}| \leqslant g(V)+e_{\mathcal{E} \cup \mathcal{A}}(\{V\})=g(V)$. This yields $f(V) \leqslant k \leqslant g(V)$.

By $(1), k-g(v) \leqslant k-f(v)$. By $f(V) \leqslant k$ and $f \geqslant 0$, it follows that $0 \leqslant k-f(V) \leqslant$ $k-f(v)$. By $(3)$ for $\mathcal{P}=\{v\}$, we obtain $k-g(v)=k|\mathcal{P}|-g(\cup \mathcal{P}) \leqslant e_{\mathcal{E} \cup \mathcal{A}}(\mathcal{P})=e_{\mathcal{E} \cup \mathcal{A}}(\{v\})=$ $d_{\mathcal{A}^{\prime}}^{-}(v)$. Then, by $0 \leqslant d_{\mathcal{A}^{\prime}}^{-}(v),(7)$ follows.

Let $\boldsymbol{V}^{\prime}=\{v \in V: k-g(v)>0\}$. This yields $\sum_{v \in V} \max \{k-g(v), 0\}=\sum_{v \in V^{\prime}}(k-$ $g(v))=k(|V|-1)-\left(g\left(V^{\prime}\right)+k\left(\left|V-V^{\prime}\right|-1\right)\right)$. If $V^{\prime} \neq V$, by $g \geqslant 0$ it follows that $g\left(V^{\prime}\right)+k\left(\left|V-V^{\prime}\right|-1\right) \geqslant g\left(V^{\prime}\right) \geqslant 0$. If $V^{\prime}=V$, by $g(V) \geqslant k$, we obtain $g\left(V^{\prime}\right)+k(\mid V-$ $\left.V^{\prime} \mid-1\right)=g(V)-k \geqslant 0$. This yields the first inequality of (8). 
Let $\boldsymbol{V}^{\prime \prime}=\left\{v \in V: k-f(v)<d_{\mathcal{A}^{\prime}}^{-}(v)\right\}$. Let $\mathcal{P}=\left\{\{v\}: v \in V-V^{\prime \prime}\right\}$. Note that any element in $\mathcal{E}(\mathcal{P}) \cup \mathcal{A}(\mathcal{P})$ provides at least one dyperedge in $\mathcal{A}^{\prime}$ entering a vertex in $V-V^{\prime \prime}$. Hence, by (2), we obtain

$$
\begin{aligned}
\sum_{v \in V} \min \left\{k-f(v), d_{\mathcal{A}^{\prime}}^{-}(v)\right\} & =\sum_{v \in V^{\prime \prime}}(k-f(v))+\sum_{v \in V-V^{\prime \prime}} d_{\mathcal{A}^{\prime}}^{-}(v) \\
& \geqslant\left(k\left|V^{\prime \prime}\right|-f\left(V^{\prime \prime}\right)\right)+e_{\mathcal{E} \cup \mathcal{A}}(\mathcal{P}) \\
& \geqslant(k(|V|-|\mathcal{P}|)-f(V-\cup \mathcal{P}))+k(|\mathcal{P}|-1)+f(V-\cup \mathcal{P}) \\
& =k(|V|-1) .
\end{aligned}
$$

It follows that the second inequality of (8) is satisfied.

We now show that Theorems 13 and 6(a) imply Theorem 8 .

Proof of Theorem 8. Necessity is proved in Lemma 5.

To see sufficiency, suppose that (1), (2) and (3) are satisfied. Let $\mathcal{D}_{\mathcal{F}}=\left(V, \mathcal{A}^{\prime}=\right.$ $\mathcal{A} \cup \mathcal{A}_{\mathcal{E}}$ ) be the directed extension of $\mathcal{F}$. By Lemma 6, (7) and (8) are satisfied. It now follows from Corollary 1 that $\mathbf{M}_{\mathcal{D}_{\mathcal{F}}}^{(k, f, g)}$ is well-defined. Again, we use $\mathbf{M}_{\mathbf{1}}$ and $\mathbf{M}_{\mathbf{2}}$ for $\mathbf{M}_{\mathcal{F}}^{k}$ and $\mathbf{M}_{\mathcal{D} \mathcal{F}}^{(k, f, g)}$, respectively. Suppose for a contradiction that no $(k, f, g)$-flexible packing of spanning mixed hyperarborescences exists in $\mathcal{F}$. By Lemma 4 , no $(k, f, g)$-feasible packing of spanning hyperarborescences exists in $\mathcal{D}_{\mathcal{F}}$. Now Theorem 13 implies that $M_{1}$ and $M_{2}$ do not have a common independent set of size $k(|V|-1)$. The next result allows to fix a certain structure leading to a contradiction later.

Claim 5. There exist a partition $\mathcal{P}$ of $V$ and $\mathcal{K} \subseteq \mathcal{E}(\mathcal{P})$ such that

$$
k(|\mathcal{P}|-1)>|\mathcal{K}|+r_{\mathrm{M}_{2}}\left(\mathcal{A}(\mathcal{P}) \cup \mathcal{A}_{\mathcal{E}(\mathcal{P})-\mathcal{K}}\right) .
$$

Proof. $\mathrm{As}_{1}$ and $\mathrm{M}_{2}$ do not have a common independent set of size $k(|V|-1)$, Theorem 6(a) implies that there exists a dyperedge set $\mathcal{Z}^{\prime} \subseteq \mathcal{A}^{\prime}$ such that $k(|V|-1)>r_{\mathrm{M}_{1}}\left(\mathcal{Z}^{\prime}\right)+$ $r_{\mathrm{M}_{2}}\left(\mathcal{A}^{\prime}-\mathcal{Z}^{\prime}\right)$. By Proposition 3, there exists a partition $\mathcal{P}$ of $V$ such that, for $\mathcal{K}=\{e \in$ $\left.\mathcal{E}(\mathcal{P}): \mathcal{Z}^{\prime} \cap \mathcal{A}_{e} \neq \varnothing\right\}$, we have $r_{\mathrm{M}_{1}}\left(\mathcal{Z}^{\prime}\right)=\left|\mathcal{Z}^{\prime} \cap \mathcal{A}(\mathcal{P})\right|+|\mathcal{K}|+k(|V|-|\mathcal{P}|)$. By subcardinality and monotonicity of $r_{\mathrm{M}_{2}}$, we have $\left|\mathcal{Z}^{\prime} \cap \mathcal{A}(\mathcal{P})\right|+r_{\mathrm{M}_{2}}\left(\mathcal{A}^{\prime}-\mathcal{Z}^{\prime}\right) \geqslant r_{\mathrm{M}_{2}}\left(\mathcal{A}^{\prime}-\left(\mathcal{Z}^{\prime}-\mathcal{A}(\mathcal{P})\right)\right) \geqslant$ $r_{\mathrm{M}_{2}}\left(\mathcal{A}(\mathcal{P}) \cup \mathcal{A}_{\mathcal{E}(\mathcal{P})-\mathcal{K}}\right)$.

The above three inequalities yield

$$
\begin{aligned}
k(|\mathcal{P}|-1) & >r_{\mathrm{M}_{1}}\left(\mathcal{Z}^{\prime}\right)+r_{\mathrm{M}_{2}}\left(\mathcal{A}^{\prime}-\mathcal{Z}^{\prime}\right)-k(|V|-|\mathcal{P}|) \\
& =\left|\mathcal{Z}^{\prime} \cap \mathcal{A}(\mathcal{P})\right|+|\mathcal{K}|+r_{\mathrm{M}_{2}}\left(\mathcal{A}^{\prime}-\mathcal{Z}^{\prime}\right) \\
& \geqslant|\mathcal{K}|+r_{\mathrm{M}_{2}}\left(\mathcal{A}(\mathcal{P}) \cup \mathcal{A}_{\mathcal{E}(\mathcal{P})-\mathcal{K})}\right.
\end{aligned}
$$

Let $\mathcal{P}$ be the partition of $V$ and $\mathcal{K}$ the hyperedge set from Claim 5 and let $\mathcal{Z}=$ $\mathcal{A}(\mathcal{P}) \cup \mathcal{A}_{\mathcal{E}(\mathcal{P})-\mathcal{K}}$. For some $X \in \mathcal{P}$, a dyperedge $a \in \mathcal{A} \cup \mathcal{A}_{\mathcal{E}-\mathcal{K}}$ contributes to either of $\sum_{v \in X} d_{\mathcal{Z}}^{-}(v)$ and $d_{\mathcal{A} \cup \mathcal{A}_{\mathcal{E}-\mathcal{K}}}(X)$ if and only if head $(a) \in X$ and tail $(a)-X \neq \varnothing$. This yields

$$
\sum_{v \in X} d_{\mathcal{Z}}^{-}(v)=d_{\mathcal{A} \cup \mathcal{A}_{\mathcal{E}-\mathcal{K}}}^{-}(X)
$$


For all $\mathcal{P}^{\prime} \subseteq \mathcal{P}$, as every hyperedge in $\mathcal{E}\left(\mathcal{P}^{\prime}\right)$ contributes to one of $|\mathcal{K}|$ and $\sum_{X \in \mathcal{P}^{\prime}} d_{\mathcal{A}_{\mathcal{E}-\mathcal{K}}}^{-}(X)$, we have

$$
|\mathcal{K}|+\sum_{X \in \mathcal{P}^{\prime}} d_{\mathcal{A} \cup \mathcal{A}_{\mathcal{E}-\mathcal{K}}}^{-}(X) \geqslant e_{\mathcal{E} \cup \mathcal{A}}\left(\mathcal{P}^{\prime}\right) .
$$

Using Corollary 1, we distinguish two cases depending on where the rank of $\mathcal{Z}$ in $\mathrm{M}_{2}$ is attained.

Case 1. $r_{\mathrm{M}_{2}}(\mathcal{Z})=\sum_{v \in V} \min \left\{k-f(v), d_{\mathcal{Z}}^{-}(v)\right\}$.

Let $\mathcal{P}^{\prime}=\left\{X \in \mathcal{P}: d_{\mathcal{Z}}^{-}(v) \leqslant k-f(v)\right.$ for all $\left.v \in X\right\}$. For all $X \in \mathcal{P}^{\prime}$, by the definition of $\mathcal{P}^{\prime}$ and (10), we have $\sum_{v \in X} \min \left\{k-f(v), d_{\mathcal{Z}}^{-}(v)\right\}=\sum_{v \in X} d_{\mathcal{Z}}^{-}(v)=d_{\mathcal{A} \cup \mathcal{A}_{\mathcal{E}-\mathcal{K}}}^{-}(X)$. For all $X \in \mathcal{P}-\mathcal{P}^{\prime}$, there exists a vertex $v_{X} \in X$ with $k-f\left(v_{X}\right)<d_{\mathcal{Z}}^{-}\left(v_{X}\right)$, and then, by $f, k-f, d_{\mathcal{Z}}^{-} \geqslant 0$, we have $k-f(X) \leqslant k-f\left(v_{X}\right)=\min \left\{k-f\left(v_{X}\right), d_{\mathcal{Z}}^{-}\left(v_{X}\right)\right\} \leqslant$ $\sum_{v \in X} \min \left\{k-f(v), d_{\mathcal{Z}}^{-}(v)\right\}$.

Then, by (9), the case distinction made, (11) and (2) for $\mathcal{P}^{\prime}$, we obtain

$$
\begin{aligned}
k(|\mathcal{P}|-1) & >|\mathcal{K}|+r_{\mathrm{M}_{2}}\left(\mathcal{A}(\mathcal{P}) \cup \mathcal{A}_{\mathcal{E}(\mathcal{P})-\mathcal{K})}\right. \\
& =|\mathcal{K}|+r_{\mathrm{M}_{2}}(\mathcal{Z}) \\
& =|\mathcal{K}|+\sum_{v \in V} \min \left\{k-f(v), d_{\mathcal{Z}}^{-}(v)\right\} \\
& =|\mathcal{K}|+\sum_{X \in \mathcal{P}^{\prime}} \sum_{v \in X} \min \left\{k-f(v), d_{\mathcal{Z}}^{-}(v)\right\}+\sum_{X \in \mathcal{P}-\mathcal{P}^{\prime}} \sum_{v \in X} \min \left\{k-f(v), d_{\mathcal{Z}}^{-}(v)\right\} \\
& \geqslant|\mathcal{K}|+\sum_{X \in \mathcal{P}^{\prime}} d_{\mathcal{A} \cup \mathcal{A}_{\mathcal{E}-\mathcal{K}}}^{-}(X)+\sum_{X \in \mathcal{P}-\mathcal{P}^{\prime}}(k-f(X)) \\
& \geqslant e_{\mathcal{E} \cup \mathcal{A}}\left(\mathcal{P}^{\prime}\right)+k\left(|\mathcal{P}|-\left|\mathcal{P}^{\prime}\right|\right)-f\left(V-\cup \mathcal{P}^{\prime}\right) \\
& \geqslant k\left(|\mathcal{P}|-\left|\mathcal{P}^{\prime}\right|\right)+k\left(\left|\mathcal{P}^{\prime}\right|-1\right) \\
& =k(|\mathcal{P}|-1)
\end{aligned}
$$

a contradiction.

Case 2. $r_{\mathrm{M}_{2}}(\mathcal{Z})=k(|V|-1)-\sum_{v \in V} \max \left\{0, k-g(v)-d_{\mathcal{Z}}^{-}(v)\right\}$.

Let $\mathcal{P}^{\prime \prime}=\left\{X \in \mathcal{P}: k-g(v)-d_{\mathcal{Z}}^{-}(v) \geqslant 0\right.$ for all $\left.v \in X\right\}$. For all $X \in \mathcal{P}^{\prime \prime}$, by the definition of $\mathcal{P}^{\prime \prime}$ and (10), we have $\sum_{v \in X} \max \left\{0, k-g(v)-d_{\mathcal{Z}}^{-}(v)\right\}=k|X|-$ $g(X)-d_{\mathcal{A} \cup \mathcal{A}_{\mathcal{E}-\mathcal{K}}}^{-}(X)$. For all $X \in \mathcal{P}-\mathcal{P}^{\prime \prime}$, there exists a vertex $v_{X} \in X$ with $0>$ $k-g\left(v_{X}\right)-d_{\mathcal{Z}}^{-}\left(v_{X}\right)$, and then, by $g, d_{\mathcal{Z}}^{-} \geqslant 0$, we have $\sum_{v \in X} \max \left\{0, k-g(v)-d_{\mathcal{Z}}^{-}(v)\right\}=$ $\sum_{v \in X-v_{X}} \max \left\{0, k-g(v)-d_{\mathcal{Z}}^{-}(v)\right\} \leqslant k(|X|-1)$. By (9) and the case distinction we made, we obtain $k(|\mathcal{P}|-1)>|\mathcal{K}|+r_{\mathrm{M}_{2}}\left(\mathcal{A}(\mathcal{P}) \cup \mathcal{A}_{\mathcal{E}(\mathcal{P})-\mathcal{K}}\right)=|\mathcal{K}|+r_{\mathrm{M}_{2}}(\mathcal{Z})=|\mathcal{K}|+k(|V|-1)-$ $\sum_{v \in V} \max \left\{0, k-g(v)-d_{\mathcal{Z}}^{-}(v)\right\}$.

This yields

$$
\begin{aligned}
k(|V|-|\mathcal{P}|)+|\mathcal{K}| & <\sum_{v \in V} \max \left\{0, k-g(v)-d_{\mathcal{Z}}^{-}(v)\right\} \\
& =\sum_{X \in \mathcal{P}^{\prime \prime}} \sum_{v \in X} \max \left\{0, k-g(v)-d_{\mathcal{Z}}^{-}(v)\right\}+\sum_{X \in \mathcal{P}-\mathcal{P}^{\prime \prime}} \sum_{v \in X} \max \left\{0, k-g(v)-d_{\mathcal{Z}}^{-}(v)\right\}
\end{aligned}
$$




$$
\begin{aligned}
& \leqslant \sum_{X \in \mathcal{P}^{\prime \prime}}\left(k|X|-g(X)-d_{\mathcal{A} \cup \mathcal{A}_{\mathcal{E}-\mathcal{K}}}^{-}(X)\right)+\sum_{X \in \mathcal{P}-\mathcal{P}^{\prime \prime}} k(|X|-1) \\
& =k\left(|V|-\left|\mathcal{P}-\mathcal{P}^{\prime \prime}\right|\right)-\sum_{X \in \mathcal{P}^{\prime \prime}}\left(g(X)+d_{\mathcal{A} \cup \mathcal{A}_{\mathcal{E}-\mathcal{K}}}^{-}(X)\right) .
\end{aligned}
$$

We obtain by (11) and (3) for $\mathcal{P}^{\prime \prime}$ that

$$
\begin{aligned}
k\left|\mathcal{P}^{\prime \prime}\right| & >|\mathcal{K}|+\sum_{X \in \mathcal{P}^{\prime \prime}} d_{\mathcal{A} \cup \mathcal{A}_{\mathcal{E}-\mathcal{K}}}^{-}(X)+\sum_{X \in \mathcal{P}^{\prime \prime}} g(X) \\
& \geqslant e_{\mathcal{A} \cup \mathcal{E}}\left(\mathcal{P}^{\prime \prime}\right)+g\left(\cup \mathcal{P}^{\prime \prime}\right) \\
& \geqslant k\left|\mathcal{P}^{\prime \prime}\right|
\end{aligned}
$$

a contradiction.

The case distinction is complete which finishes the proof of Theorem 8 .

Finally, we deal with the algorithmic consequences of Theorem 13 which are contained in Theorem 9.

Proof of Theorem 9. It can be checked efficiently whether (7) and (8) are satisfied. If not, then, by Lemma 6, one of (1), (2) and (3) is not satisfied. By Lemma 5 , no $(k, f, g)$ flexible packing of spanning mixed hyperarborescences exists in $\mathcal{F}$. Otherwise, it follows from Corollary 1 that $\mathrm{M}_{\mathcal{D}_{\mathcal{F}}}^{(k, f, g)}$ is well-defined and from Theorem 13 that the common independent sets of $\mathrm{M}_{\mathcal{F}}^{k}$ and $\mathrm{M}_{\mathcal{D}_{\mathcal{F}}}^{(k, f, g)}$ of size $k(|V|-1)$ are exactly the dyperedge sets of the $(k, f, g)$-feasible packings of spanning hyperarborescences in $\mathcal{D}_{\mathcal{F}}$.

Define $\boldsymbol{w}^{\prime}: \mathcal{A} \cup \mathcal{A}_{\mathcal{E}} \rightarrow \mathbb{R}$ by $w^{\prime}(a)=w(a)$ for all $a \in \mathcal{A}$ and $w^{\prime}(a)=w(e)$ for all $a \in \mathcal{A}_{e}$ for all $e \in \mathcal{E}$. We first check if there is a common independent set of $\mathrm{M}_{\mathcal{F}}^{k}$ and $\mathrm{M}_{\mathcal{D} \mathcal{F}}^{(k, f, g)}$ of size $k(|V|-1)$ and if this is the case, we find a common independent set $\mathcal{A}^{*}$ of $\mathrm{M}_{\mathcal{F}}^{k}$ and $\mathrm{M}_{\mathcal{D} \mathcal{F}}^{(k, f, g)}$ of size $k(|V|-1)$ minimizing $w^{\prime}\left(\mathcal{A}^{*}\right)$. This can be done in polynomial time using Theorem $6(\mathrm{~b})$ because polynomial time independence oracles for $\mathrm{M}_{\mathcal{F}}^{k}$ and $\mathrm{M}_{\mathcal{D}_{\mathcal{F}}}^{(k, f, g)}$ are available by Lemmas 2 and 3 . Now consider the subdypergraph $\mathcal{D}^{*}=\left(V, \mathcal{A}^{*}\right)$ of $\mathcal{D}_{\mathcal{F}}$ and let $\boldsymbol{R}$ be the multiset in $V$ in which every $v \in V$ is contained $k-d_{\mathcal{A}^{*}}^{-}(v)$ times. By the definition $\mathrm{M}_{\mathcal{D}_{\mathcal{F}}}^{(k, f, g)}$, we have $k-d_{\mathcal{A}^{*}}^{-}(v) \geqslant f(v) \geqslant 0$ for all $v \in V$, so $R$ is well-defined. By Theorem $5, \mathcal{A}^{*}$ can be decomposed into the dyperedge sets of a packing of spanning hyperarborescences of $\mathcal{D}_{\mathcal{F}}$ with root set $R$ in polynomial time. Replacing dyperedges in the packing in $\mathcal{A}_{e}$ by $e$ for all $e \in \mathcal{E}$, we obtain, by Lemma 4 , the desired packing in $\mathcal{F}$.

\section{References}

[1] J. Edmonds, Matroid Partition, Mathematics of the Decision Sciences: Part 1 (G.B. Dantzig and A.F. Veinott, eds.), American Mathematical Society, 335-345, 1968.

[2] J. Edmonds, Submodular functions, matroids, and certain polyhedra, in: eds. R. Guy, H. Hanani, N. Sauer, and J. Schönheim, Combinatorial Structures and their Applications, Gordon and Breach, New York, 69-70, 1970. 
[3] J. Edmonds, Edge-disjoint branchings, in: B. Rustin (ed.), Combinatorial Algorithms, Academic Press, New York, 91-96, 1973.

[4] J. Edmonds, Some well-solved problems in combinatorial optimization, in: Combinatorial Programming: Methods and Applications (Proceedings NATO Advanced Study Institute, Versailles, 1974. B. Roy, ed.), Reidel, Dordrecht, 285-301, 1975.

[5] J. Edmonds, Matroid intersection, Ann. Disc. Math. 4, 39-49, 1979.

[6] Q. Fortier, Cs. Király, M. Léonard, Z. Szigeti, A. Talon, Old and new results on packing arborescences in dypergraphs, Discrete Appl. Math. 242, 26-33, 2018.

[7] A. Frank, On disjoint trees and arborescences. In Algebraic methods in graph theory, 25, Colloquia Mathematica Soc. J. Bolyai, North-Holland, 159-169, 1978.

[8] A. Frank, Gráfelmélet, https://web.cs.elte.hu/ frank/jegyzet/graf/graf. 2014.pdf

[9] A. Frank, T. Király, Z. Király, On the orientation of graphs and hypergraphs, Discrete Appl. Math. 131(2), 385-400, 2003.

[10] A. Frank, T. Király, M. Kriesell, On decomposing a hypergraph into $k$ connected sub-hypergraphs, Discrete Appl. Math. 131, 373-383, 2003.

[11] A. Frank, Connections in Combinatorial Optimization, Oxford University Press, 2011.

[12] H. Gao, D. Yang, Packing of spanning mixed arborescences, https://arxiv.org/ abs $/ 2005.03218$

[13] M. Lorea, Hypergraphes et matroides, Cahiers Centre Etudes Rech. Oper. 17, 289291, 1975.

[14] Z. Szigeti, A survey on arborescence packings, unpublished manuscript. 